\title{
Ballistic Annihilation
}

\author{
Paul L. Kaprivsky ${ }^{1,2}$ and Clément Sire ${ }^{1}$ \\ ${ }^{1}$ Laboratoire de Physique Quantique (UMR C5626 du CNRS), Université Paul Sabatier, 31062 Toulouse Cedex, France \\ 2 Center for Polymer Physics and Department of Physics, Boston University, Boston, MA 02215, USA
}

\begin{abstract}
Ballistic annihilation with continuous initial velocity distributions is investigated in the framework of Boltzmann equation. The particle density and the rms velocity decay as $c \sim t^{-\alpha}$ and $\langle v\rangle \sim t^{-\beta}$, with the exponents depending on the initial velocity distribution and the spatial dimension. For instance, in one dimension for the uniform initial velocity distribution we find $\beta=0.230472 \ldots$. We also solve the Boltzmann equation for Maxwell particles and very hard particles in arbitrary spatial dimension. These solvable cases provide bounds for the decay exponents of the hard sphere gas.
\end{abstract}

PACS numbers: 05.20.Dd, 03.20.+i, $82.20 \mathrm{Mj}$

Ballistic annihilation is the kinetic process which involves particles undergoing ballistic motion and annihilating upon colliding. Ballistic annihilation underlies numerous apparently unrelated phenomena, e.g., growth and coarsening processes and traffic flows [1] 1 . In coarsening processes, for instance, domain walls have a natural particle interpretation. Despite all that, little is known on irreversible processes where the reactants move ballistically while the contrasting situation of diffusioncontrolled processes is well understood [5].

There exist few theoretical results on ballistic annihilation in one dimension with discrete velocity distributions. For the simplest binary velocity distribution, the ballistic annihilation process has been solved in one dimension by Elskens and Frisch [6], see also Refs. [1,7]9; some analytical results are also available for the ternary velocity distribution 10,11. No solutions have been found for continuous initial velocity distributions, although the decay exponents have been determined numerically [12,13]. This lack of analytical results is especially striking given that ballistic aggregation processes admit exact solutions in one dimension for arbitrary initial velocity distributions 14, 15.

In this work, we consider ballistic annihilation with continuous isotropic initial velocity distributions in arbitrary dimension. Our analysis is performed in the framework of Boltzmann equation approach. This scheme involves an uncontrolled approximation and generally leads to erroneous results for ballistic annihilation with discrete velocity distributions. For continuous velocity distributions, however, the decay exponents found from numerical integration of the Boltzmann equation 12 are an excellent agreement with simulation results [12,13]. Furthermore, for solvable one dimension ballistic aggregation processes with continuous velocity distributions [14, 1.5], the decay exponents computed from the Boltzmann equation are exact; for higher-dimensional ballistic aggregation process [16], the decay exponents determined within the Boltzmann framework [16,17] appear to be exact as well. Therefore, we believe that the Boltzmann equation approach provides exact decay exponents for ballistic annihilation with continuous velocity distributions.

For clarity, we start with one-dimensional ballistic an- nihilation process. An appropriate Boltzmann equation reads 12$]$

$$
\frac{\partial P(v, t)}{\partial t}=-P(v, t) \int_{-\infty}^{\infty} d v^{\prime}\left|v-v^{\prime}\right| P\left(v^{\prime}, t\right)
$$

In the long time limit, the velocity distribution approaches a scaling form

$$
P(v, t)=t^{\beta-\alpha} F(x), \quad \text { with } \quad x=v t^{\beta} .
$$

The two basic exponents determine the behavior of the particle concentration $c(t)$ and the rms velocity $\langle v\rangle$ :

$$
c(t) \sim t^{-\alpha}, \quad\langle v\rangle \sim t^{-\beta} .
$$

Formally, $c$ and $\langle v\rangle$ are defined as follows

$$
\begin{aligned}
& c(t)=\int_{-\infty}^{\infty} d v P(v, t)=t^{-\alpha} \int_{-\infty}^{\infty} d x F(x), \\
& \langle v\rangle^{2}=c^{-1} \int_{-\infty}^{\infty} d v v^{2} P(v, t)=t^{-2 \beta} \frac{\int_{-\infty}^{\infty} d x x^{2} F(x)}{\int_{-\infty}^{\infty} d x F(x)} .
\end{aligned}
$$

By inserting the scaling form (2) into Eq. (11) one finds $\alpha+\beta=1$ and an equation for the scaling function

$$
2 \beta-1+\beta x \frac{F^{\prime}(x)}{F(x)}=-\int_{-\infty}^{\infty} d x^{\prime}\left|x-x^{\prime}\right| F\left(x^{\prime}\right) .
$$

In the following, we always consider isotropic initial velocity distributions. In one dimension, this requirement reads $P_{0}(v)=P_{0}(-v)$ and it implies the symmetry for later times $P(v, t)=P(-v, t)$ and the symmetry of the scaling function $F(x)=F(-x)$.

The large $x$ behavior of the scaled velocity distribution is found by noting that in this region the integral on the right-hand side of Eq. (任) simplifies to $C x$, where $C$ is the normalization constant, $C=\int_{-\infty}^{\infty} d x F(x)$. Solving the resulting differential equation gives

$$
F(x) \sim x^{(1-2 \beta) / \beta} e^{-C x / \beta} \text { when } x \rightarrow \infty .
$$

For a given $\beta$, a solution of Eq. (4) would not agree with the boundary condition at $x=0$ which is implied 
by the initial velocity distribution. Thus, we arrive at an eigenvalue problem. For instance, we should require $F^{\prime}(0)=0$ if the initial velocity distribution is flat near the origin. Solving this eigenvalue problem numerically gives $\beta_{\text {flat }}=0.230472 \ldots$, to be compared with $\beta_{\text {flat }} \approx 0.22$ [12] and $\beta_{\text {flat }} \approx 0.19$ [13] found from Monte Carlo simulations. Similarly, for the initial velocity distribution satisfying $P_{0}(v) \sim|v|$ we find $\beta_{\text {linear }}=0.166649 \ldots$

We now outline an approximate analytical computation of the exponent $\beta$. First we note that Eq. (4) can be reduced to an ordinary differential equation after a double differentiation. This equation can be further simplified by the rescaling $x \rightarrow x \sqrt{\beta}$ that eliminates the $\beta$ factor. The governing equation then reads

$$
x f^{\prime \prime \prime}+2 f^{\prime \prime}=2 \exp (-f),
$$

with $f(x)=-\ln F(x)$. We shall also use the relation

$$
\int_{0}^{\infty} d x x F(x)=(2 \beta)^{-1}-1
$$

which will play the role of a normalization condition. Equation (7) is just Eq. (4) at $x=0$.

Although Eq. (5) cannot be solved exactly, an approximate solution $f_{1}$ can be found by replacing the right-hand side by $e^{-f_{0}}$, where $f_{0}$ is a reasonable approximation for $f$. In principle, this approximation scheme can be repeated again starting from $f_{1}$, and should finally lead to the exact form for $f$. As the starting point, we choose

$$
F_{0}(x)=e^{-f_{0}(x)}=\left(1+\frac{x}{\nu}+\frac{1-\nu^{2}}{2 \nu^{2}} x^{2}\right) e^{-x / \nu} .
$$

The function $F_{0}(x)$ is constructed in such a way, that the small $x$ expansion is correct up to the second order. Additionally, $F_{0}(x)$ exhibits an exponential decay for large $x$, in agreement with the exact asymptotic behavior (5). The parameter $\nu$ is yet to be determined. Replacing now the right-hand side of Eq. (6) by $F_{0}(x)$ from Eq. (8) and solving the resulting linear differential equation gives

$$
\begin{aligned}
f_{1}(x) & =2 \nu\left(3-\nu^{2}\right) x+\nu^{2}\left(7-5 \nu^{2}\right)\left(1-e^{-x / \nu}\right) \\
& -\nu\left(1-\nu^{2}\right) x e^{-x / \nu} \\
& -6 \nu^{2}\left(2-\nu^{2}\right) \int_{0}^{x / \nu} \frac{1-\exp (-t)}{t} d t .
\end{aligned}
$$

The constant $\nu$ can now be calculated self-consistently by imposing the constraint

$$
\nu^{-1}=2 \int_{0}^{\infty} F(x) d x
$$

which can be obtained by integrating Eq. (6). Plugging $F_{\text {appr }}=e^{-f_{1}(x)}$ into Eq. (10) gives $\nu_{\text {appr }}^{-1} \cong 2.67156$ to be compared to the exact numerical value $\nu^{-1}=2.65826 \ldots$ Finally, Eq. (7) leads to $\beta_{\text {appr }} \cong 0.22898$, in good agreement with the exact value obtained above. In Fig. 1, we plot the exact numerical scaling function $F$ and the approximate solution $F_{\text {appr }}=e^{-f_{1}(x)}$ with $f_{1}(x)$ given by Eq. (9). The approximate solution is extremely good as the relative error is always less than $0.5 \%$.

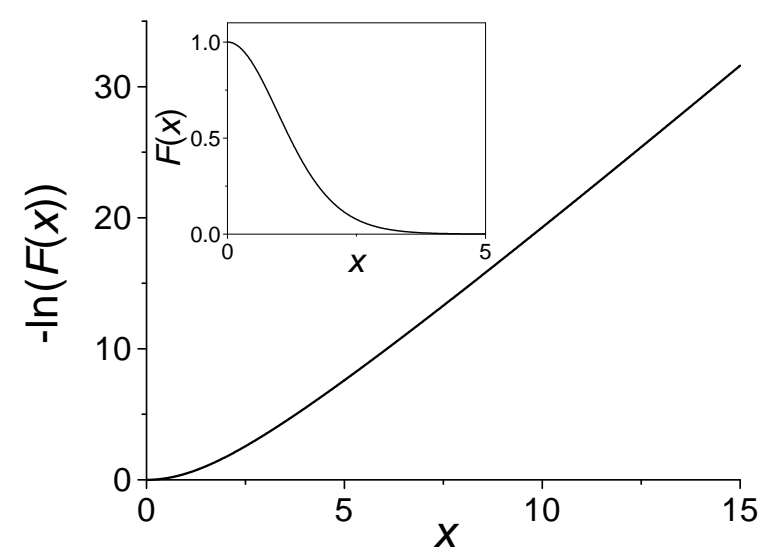

FIG. 1. Plot on a semi-log scale of the scaling function $F$ and its approximation given by Eq. (9). The insert shows both functions in normal scale. Both scaling functions are indistinguishable as the relative error is less than $0.5 \%$

In higher dimensions, the Boltzmann equation reads

$$
\frac{\partial P(\mathbf{v}, t)}{\partial t}=-P(\mathbf{v}, t) \int d \mathbf{w}|\mathbf{v}-\mathbf{w}| P(\mathbf{w}, t) .
$$

For isotropic initial velocity distributions, the appropriate scaling variable is $x=v t^{\beta}$ with $v=|\mathbf{v}|$, and the scaling form is $P(\mathbf{v}, t)=t^{d \beta-\alpha} F(x)$. Plugging this scaling form into Eq. (11) and using the spherical coordinates to simplify the collision integral we obtain

$$
\begin{aligned}
(d+1) \beta & -1+\beta x \frac{F^{\prime}(x)}{F(x)} \\
& =-\Omega_{d-1} \int_{0}^{\infty} d y y^{d-1} E_{d}(x, y) F(y) .
\end{aligned}
$$

Here $\Omega_{d-1}$ is the surface area of the unit sphere in $d-1$ dimensions and

$$
E_{d}(x, y)=\int_{0}^{\pi} d \phi(\sin \phi)^{d-2} \sqrt{x^{2}+y^{2}-2 x y \cos \phi}
$$

In two dimensions, $E_{2}(x, y)=2(x+y) E(k)$, where $E(k)$ is the complete elliptic integral of the second kind with modulus $k=\frac{2 \sqrt{x y}}{x+y}$. Similarly, in an arbitrary even dimension $E_{d}(x, y)$ can be expressed via elliptic integrals. In odd dimensions, $E_{d}(x, y)$ can be expressed in terms of elementary functions. In the most interesting three dimensional case, one reduces Eq. (12) to

$$
\begin{aligned}
4 \beta & -1+\beta x \frac{F^{\prime}(x)}{F(x)} \\
& =-\frac{2 \pi}{3} \int_{0}^{\infty} d y y \frac{(x+y)^{3}-|x-y|^{3}}{x} F(y) .
\end{aligned}
$$


One should solve Eq. (12) subject to an appropriate boundary condition at the origin. Overall, the task reduces again to the eigenvalue problem. Note that the approximation scheme explicitly presented above in $d=1$ could be equally applied in higher dimensions.

Thus, the decay exponents can be determined with arbitrarily high precision in arbitrary spatial dimension for arbitrary isotropic initial velocity distribution. This method, however, does not solve the Boltzmann equation (11). We now provide two approximate solutions to the Boltzmann equation for arbitrary initial conditions and arbitrary spatial dimension $d$. These solutions are in fact exact solutions of Boltzmann equations with collision kernels $\sigma(g)$ different from $\sigma(g)=g$ characterizing the hard sphere gas (here $g \equiv|\mathbf{v}-\mathbf{w}|$ is the relative velocity). Note that such collision kernels naturally arise in kinetic theory of interacting particles [18]. In the present context, the generalized Boltzmann equation reads

$$
\frac{\partial P(\mathbf{v}, t)}{\partial t}=-P(\mathbf{v}, t) \int d \mathbf{w} \sigma(g) P(\mathbf{w}, t) .
$$

Let us compare dimensions of the left and right-hand side of Eq. (15). The velocity distribution has dimension $[P]=T / L^{d+1}$ which implies $[\sigma]=L^{d} / T$; therefore $[\sigma(g) / g]=L^{d-1}$. The remaining quantity with dimension of length, the "interaction" radius, should be extracted from the collision process. For hard sphere gas, the relevant interaction radius is simply the geometrical radius $a$ of the spheres, so $\sigma(g)=g a^{d-1}$. The constant factor $a^{d-1}$ can be absorbed into the time variable - this is what we have done in Eq. (11). For particles interacting through a two-body power law potential, $U(r) \propto r^{-n}$, the energy conservation implies $g^{2} \sim r^{-n}$. Thus, $\sigma(g) \sim g r^{d-1} \sim g^{\nu}$ with $\nu=1-\frac{2(d-1)}{n}$. The hard sphere gas $(\nu=1)$ is recovered for $n=\infty$. The velocity independent kernel $(\nu=0)$, the so-called "Maxwell" gas, arises when particles interact through the power law potential with the exponent $n=2(d-1)$. When $\nu>1$, the interaction is "harder" than in the hard sphere gas (though such behavior does not arise from a simple power-law interaction potential). One particularly tractable model corresponds to $\sigma(g)=g^{2}$, the so-called gas of very hard particles 19 .

To provide a faithful analog of the original hard sphere gas, we replace $\sigma(g)=g$ by $\langle v\rangle$ in the Maxwell case and by the factor $g^{2} /\langle v\rangle$ for the very hard particles. Hence for the Maxwell gas the Boltzmann equation (15) becomes

$$
\frac{\partial P(\mathbf{v}, t)}{\partial t}=-P(\mathbf{v}, t) c(t)\langle v(t)\rangle .
$$

Thus we get effectively non-interacting particles as different velocities remain uncoupled. Solving (16) yields

$$
P(\mathbf{v}, t)=c(t) P_{0}(\mathbf{v}), \quad c(t)=\frac{1}{1+\langle v\rangle t} .
$$

The moments of the velocity do not change with time, e.g., $\langle v(t)\rangle=\langle v\rangle_{0}$, and thence $\alpha=1$ and $\beta=0$.
More interesting results are found for the very hard particles. The corresponding Boltzmann equation, i.e., Eq. (15) with $\sigma(g)=g^{2} /\langle v\rangle$, can be simplified by absorbing the $\langle v\rangle^{-1}$ factor into the time variable,

$$
\tau=\int_{0}^{t} \frac{d t^{\prime}}{\left\langle v\left(t^{\prime}\right)\right\rangle}
$$

and reducing the collision integral into a combination of the moments $M_{j}(\tau)=\int d \mathbf{w} w^{j} P(\mathbf{w}, \tau)$ of the velocity distribution. The Boltzmann equation becomes

$$
\frac{\partial P(\mathbf{v}, \tau)}{\partial \tau}=-P(\mathbf{v}, \tau)\left[v^{2} M_{0}(\tau)+M_{2}(\tau)\right],
$$

and easily solved to give

$$
P(\mathbf{v}, \tau)=P_{0}(\mathbf{v}) e^{-v^{2} L_{0}(\tau)-L_{2}(\tau)} .
$$

Here $L_{j}(\tau)=\int_{0}^{\tau} d \tau^{\prime} M_{j}\left(\tau^{\prime}\right)$

To derive explicit results, it is natural to consider initial velocity distributions algebraic near the origin. To simplify algebra, we specifically choose

$$
P_{0}(\mathbf{v})=\frac{2 v^{\mu} e^{-v^{2}}}{\Omega_{d} \Gamma[(\mu+d) / 2]},
$$

where $\Omega_{d}=2 \pi^{d / 2} / \Gamma(d / 2)$ is the surface area of the unit sphere in $d$ dimensions, and the prefactor in (21) is chosen to set the initial density to unity. Combining (20) and (21) we can explicitly compute $M_{0}$ and $M_{2}$. Using then $M_{j}=\frac{d L_{j}}{d \tau}$ closes the problem. We find

$$
\frac{d L_{0}}{d \tau}=\frac{e^{-L_{2}}}{\left(1+L_{0}\right)^{\frac{\mu+d}{2}}}, \quad \frac{d L_{2}}{d \tau}=\frac{\mu+d}{2} \frac{e^{-L_{2}}}{\left(1+L_{0}\right)^{\frac{\mu+d+2}{2}}} .
$$

Solving these equations subject to $L_{0}(0)=L_{2}(0)=0$ yields

$$
\begin{aligned}
& L_{0}=[1+(\mu+d+1) \tau]^{\frac{1}{\mu+d+1}}-1, \\
& L_{2}=\frac{\mu+d}{2(\mu+d+1)} \ln [1+(\mu+d+1) \tau] .
\end{aligned}
$$

Now we compute $c=M_{0}=\frac{d L_{0}}{d \tau}$ to find the density,

$$
c=[1+(\mu+d+1) \tau]^{-\frac{\mu+d}{\mu+d+1},}
$$

and $\langle v\rangle^{2}=\frac{M_{2}}{M_{0}}=\frac{\mu+d}{2}\left(1+L_{0}\right)^{-1}$,

$$
\langle v\rangle=\sqrt{\frac{\mu+d}{2}}[1+(\mu+d+1) \tau]^{-\frac{1}{2(\mu+d+1)}} .
$$

By inserting Eq. (23) into Eq. (18) we can express $\tau$ via the original time variable $t$. Finally, we arrive at

$$
\alpha=\frac{2(\mu+d)}{2(\mu+d)+1}, \quad \beta=\frac{1}{2(\mu+d)+1} .
$$


Note that the exponent relation $\alpha+\beta=1$ which is valid for any $\mu$ and $d$. This sum rule immediately follows from an elementary mean-free path argument: $a^{d-1} c\langle v\rangle t \sim 1$.

The above exact values of the exponents in the two solvable limits appear to provide the strict bounds for the hard sphere case:

$$
\frac{2(\mu+d)}{2(\mu+d)+1}<\alpha<1, \quad 0<\beta<\frac{1}{2(\mu+d)+1} .
$$

These bounds are fair for small $d$ and they get more and more stringent as the spatial dimension increases.

The above method of solving the Boltzmann equation can be adapted to the more general collision kernels $\sigma\left(\mathbf{v}, \mathbf{v}^{\prime}\right)=\langle v\rangle^{1-\kappa}\left(|\mathbf{v}|^{\kappa}+\left|\mathbf{v}^{\prime}\right|^{\kappa}\right)$ (the most natural case corresponds to $\kappa=1)$. For $\kappa>0$, the decay exponents are given by Eqs. (24). The collision kernels $\sigma(g)=g^{2 n}$ constitute a more perspective generalization. One could try to solve the Boltzmann equation when $n$ is integer and then perform an analytic continuation to $n=1 / 2$ corresponding to the hard sphere gas. Besides the cases of Maxwell and very hard particles $(n=0$ and 1 , respectively), it is possible to work out the case of $n=2$. Unfortunately, we have not succeeded beyond that.

In the limit $d \rightarrow \infty$, the Boltzmann equation (11) becomes tractable. First of all, the collision kernel simplifies to $\sqrt{v^{2}+w^{2}}$ as (different) vectors are orthogonal in infinite dimensions. The scaled Boltzmann equation reads

$$
1-B-\beta x \frac{F^{\prime}}{F}=\Omega_{d} \int_{0}^{\infty} d y y^{d-1} \sqrt{x^{2}+y^{2}} F(y),
$$

with $B=(d+1) \beta$. The bounds of Eq. (25) lead to $\beta \rightarrow 0$ but remain non-trivial for $B, 0<B<1 / 2$. The righthand side of Eq. (26) is computed by the saddle point technique to find $C \sqrt{x^{2}+y_{*}^{2}}$, where $C$ is the normalization factor $C=\Omega_{d} \int_{0}^{\infty} d y y^{d-1} F(y)$, and $y_{*}$ is the saddle point which is found from $y_{*} F^{\prime}\left(y_{*}\right) / F\left(y_{*}\right)+d-1=0$. Hence, at the saddle point Eq. (26) gives $1=C y_{*} \sqrt{2}$. Near the origin, $F(x) \sim x^{\mu}$ and thus $\beta x F^{\prime} / F=\beta \mu \rightarrow 0$, so we find $1-B=C y_{*}=1 / \sqrt{2}$. Thus we arrive at the universal asymptotics,

$$
\beta \simeq\left(1-\frac{1}{\sqrt{2}}\right) d^{-1} \text { when } \quad d \rightarrow \infty,
$$

independently on $\mu$.

In this work, we reduced the determination of the decay exponents for ballistic annihilation to an eigenvalue problem. We found that the exponents have very nontrivial values even for the simplest initial velocity distributions. Our approach manifestly demonstrates that the decay exponents are affected only by the spatial dimension $d$ and by the exponent $\mu$ characterizing the initial velocity distribution in the $|\mathbf{v}| \rightarrow 0$ limit: $P_{0}(\mathbf{v}) \sim|\mathbf{v}|^{\mu}$. We also solved the Boltzmann equation for the Maxwell particles and very hard particles in arbitrary spatial dimension. For the hard sphere gas, we found the asymptotic behavior of the exponents in the $d \rightarrow \infty$ limit.

Interesting discussions with Eli Ben-Naim and Satya Majumdar are gratefully acknowledged. PLK also acknowledges support from NSF, ARO, and CNRS.

[1] J. Krug and H. Spohn, Phys. Rev. A 38, 4271 (1988).

[2] K. Sekimoto, Int. J. Mod. Phys. B 5, 1843 (1991).

[3] L. Frachebourg, P. L. Krapivsky, and E. Ben-Naim, Phys. Rev. Lett. 77, 2125 (1996); Phys. Rev. E 54, 6186 (1996).

[4] M. Gerwinski and J. Krug, Phys. Rev. E 60, 188 (1999).

[5] A review of diffusion-controlled processes is given in Nonequilibrium Statistical Physics in One Dimension, ed. V. Privman (Cambridge University Press, Cambridge, 1997).

[6] Y. Elskens and H. L. Frisch, Phys. Rev. A 31, 3812 (1985).

[7] V. Belitsky and P. A. Ferrari, J. Stat. Phys. 80, 517 (1995).

[8] L. Frachebourg and P. L. Krapivsky, Phys. Rev. E 55, 252 (1997).

[9] Several generalization of the binary ballistic annihilation process are discussed in E. Ben-Naim, S. Redner, and P. L. Krapivsky, J. Phys. A 29, L561 (1996); M. J. E. Richardson, J. Stat. Phys. 89, 777 (1997); Y. Kafri, J. Phys. A 33, 2365 (2000); R. A. Blythe, M. R. Evans, and Y. Kafri, cond-mat/000507\%.

[10] P. L. Krapivsky, S. Redner, and F. Leyvraz, Phys. Rev. E 51, 3977 (1995).

[11] J. Piasecki, Phys. Rev. E 51, 5535 (1995); M. Droz, P.A. Rey, L. Frachebourg, and J. Piasecki, Phys. Rev. E 51, 5541 (1995).

[12] E. Ben-Naim, S. Redner, and F. Leyvraz, Phys. Rev. Lett. 70, 1890 (1993).

[13] P.-A. Rey, M. Droz, and J. Piasecki, Phys. Rev. E 57, 138 (1998).

[14] E. Ben-Naim, P. L. Krapivsky, and S. Redner, Phys. Rev. E 50, 822 (1994).

[15] L. Frachebourg, Phys. Rev. Lett. 82, 1502 (1999).

[16] G. F. Carnevale, Y. Pomeau, and W. R. Young, Phys. Rev. Lett. 64, 2913 (1990).

[17] P. L. Krapivsky and E. Ben-Naim, Phys. Rev. E 53, 291 (1996).

[18] P. Résibois and M. de Leener, Classical Kinetic Theory of Fluids (John Wiley, New York, 1977).

[19] M. H. Ernst, Phys. Reports 78, 1 (1981). 